\title{
Love Wave Propagation in Porous Rigid Layer Lying over an Initially Stressed Half-Space
}

\author{
S. Kundu, S. Gupta, A. Chattopadhyay, and D. K. Majhi
}

\begin{abstract}
This paper devotes to study the propagation of Love waves in a fluid saturated, anisotropic, porous rigid layer lying over a prestressed, non-homogeneous, elastic half space. The dispersion equation of phase velocity is obtained. We found that the phase velocity of Love waves is considerably influenced by rigidity, porosity and anisotropy of the porous layer, inhomogeneity of the half-space and prestressing present in the media, the layer and the half-space. As a particular case dispersion equation of Love wave in rigid boundary does not coincides with that of in free bounray. Dispersion curves are plotted for different variation in inhomogeneity parameters and initial stress parameters the effect of the medium characteristics on the propagation of Love waves is discussed.
\end{abstract}

Index Terms - Love wave, anisotropic, porous rigid layer, initial stress.

\section{INTRODUCTION}

The propagation of waves in layered media is of central interest to the theoretical seismologists. A.E.H. Love [1] described a mathematical model of surface waves known as Love waves in 1911. The study of propagation of elastic waves in a statically isotropic, fluid saturated, porous solid has been first discussed by Biot ([2]-[5]). Using the Biot's theory, several investigators have studied extensively the propagation of surface waves such as Rayleigh and Love waves. Initial stresses have much effect on the propagation of elastic waves (Biot, [6]). Bose, [7] and Burridge and Vargas, [8] have contributed their work in this field.. The medium is often porous and liquid filled. The role of pore-water in seismology has been emphasized in many studies. Chattopadhyay and De [9] have studied the propagation of Love waves in a porous layer underlain by isotropic elastic medium with a rectangular irregularity at the interface. Nowinski [10] has shown the effect of high initial stresses on Love waves in an isotropic, elastic, incompressible medium. The most satisfactory theory has been given by Weiskopf [11], who considered the medium to have a particular kind of transverse-isotropy. Furthermore, the earth is an initially stressed medium. Since the composition of the Earth is heterogeneous, including very hard layers, medium heterogeneity and rigid interfaces play a significant role in the propagation of seismic waves. It is, therefore, interesting to study the Love wave propagation in a medium which is formed by an initially stressed, anisotropic, porous rigid layer lying over an elastic half-space under initial stress. The

Manuscript received September 25, 2012; revised January 13, 2013.

S. Kundu is with the Indian School of Mines, Dhanbad, India (e-mail: kundu_santi@yahoo.co.in). half-space could be nonhomogeneous because non-homogeneity characteristics is one of the most generalized elastic condition inside the earth.

\section{Formulation OF THE PROBLEM}

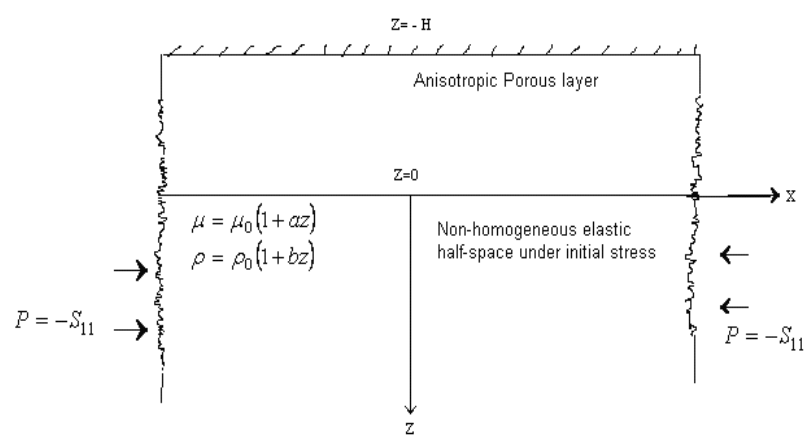

Fig. 1. Geometry of the problem.

Assume a water saturated porous rigid layer of thickness $H$ with anisotropy of the Weiskopf type along the direction of $x$ over a non-homogeneous elastic half space under the compressive initial stress $P=-S_{11}$. The surface of contact is the plane $z=0$, and the $z$-axis is directed vertically downwards. The wave is assumed to propagate along the $x$-direction.

\section{SOLUTION FOR THE UPPER LAYER}

$$
v^{\prime}=\left(A e^{i q^{\prime} z}+B e^{-i q^{\prime} z}\right) e^{i K(x-c t)}, \quad-H \leq z \leq 0
$$

where

$$
\mathrm{q}^{\prime}=K \sqrt{\frac{c^{2} d^{\prime}-\left(N-P^{\prime} / 2\right)}{L}}=K \sqrt{\gamma d\left\{\frac{c^{2}}{\frac{N}{d^{\prime}} \cdot d}-\frac{1}{d}\right\}}
$$

\section{SOlution FOR THE HALF-SPACE}

Hence the displacement component $v$ in the heterogeneous medium is given by

$$
\begin{aligned}
v= & V(z) e^{i K(x-c t)} \\
= & \frac{\mathrm{D}_{1} W_{R / 2,0}(\eta)}{(1+a z)^{\frac{1}{2}}} e^{i K(x-c t)}
\end{aligned}
$$


Expanding Whittaker's function up to linear terms eqn. reduces to

$$
v=D_{1} e^{\frac{-\gamma K(1+a z)}{a}}\left\{\frac{2 \gamma_{1} K}{a}\right\}^{1 / 2}\left[1+(1-R) \frac{\gamma_{1} K}{a}(1+a z)\right] e^{i K(x-c t)}
$$

\section{BOUNDARY CONDITIONS AND DISPERSIVE EQUATION}

The following boundary conditions are to be satisfied by solutions

$$
\begin{aligned}
& v^{\prime}=0 \text { at } z=-H \\
& v^{\prime}=v \quad \text { at } z=0 \\
& \Delta f_{y}^{\prime}=\Delta f_{y} \text { at } z=0
\end{aligned}
$$

Eliminating $A, B$ and $D_{1}$ from eqns. (1) and (3) we get

$$
\cot \left(q^{\prime} H\right)=\frac{1}{q^{\prime}} \frac{\mu_{0}}{L}\left[\frac{(1-R) \gamma_{1} K}{1+(1-R) \frac{\gamma_{1} K}{a}}-\gamma_{1} K\right]
$$

where $\gamma_{1}=\left[1-\frac{\xi}{1+a z}-\frac{c^{2}}{c_{o s}^{2}} \frac{b}{a}\right]^{1 / 2}, \xi=\frac{\mathrm{P}}{2 \mu_{0}}, \quad c$ is the phase velocity of Love waves and $R=\frac{\omega^{2}(a-b)}{c_{O S}^{2} a^{2} \gamma_{1} K}$.

\section{Particular CASES}

1) In the case when both the layer and half-space are free from initial stresses, i.e. $\xi=0$, the dispersive equation (4) takes the form

$$
\cot \left(q_{1}^{\prime} H\right)=\frac{1}{q^{\prime}} \frac{\mu_{0}}{L}\left[\frac{(1-R) \gamma_{2} K}{1+(1-R) \frac{\gamma_{2} K}{a}}-\gamma_{2} K\right]
$$

where $\gamma_{2}=\left[1-\frac{c^{2}}{c_{o s}^{2}} \frac{b}{a}\right]^{1 / 2}$ and $q_{1}^{\prime}=K \sqrt{\gamma d\left\{\frac{c^{2}}{\beta_{a}^{2}}-\frac{1}{d}\right\}}$,

2) In the case when the layer is non-porous, i.e. $f=0$, then $d=1$, then the dispersion equation (4) takes the form

$$
\cot \left(q_{2}^{\prime} H\right)=\frac{1}{q_{2}^{\prime}} \frac{\mu_{0}}{L}\left[\frac{(1-R) \gamma_{1} K}{1+(1-R) \frac{\gamma_{1} K}{a}}-\gamma_{1} K\right]
$$

where $q_{2}^{\prime}=K \sqrt{\gamma d\left\{\frac{c^{2}}{\beta_{a}^{2}}-1\right\}}$,

This is dispersive equation of Love waves in an initially stressed, non-porous anisotropic medium. 3) If the lower half-space is homogeneous, i.e. $a \rightarrow 0, \mathrm{~b} \rightarrow 0$ then the Eq. (4) is reduced to

$$
\left.\cot \left\{\gamma\left\{\frac{c^{2}}{\beta_{a}^{2}}-1\right\}\right]^{1 / 2} k H\right\}=\frac{\mu_{0}}{L} \frac{\left[\frac{c^{2}}{c_{o s}^{2}}-(1-\xi)\right]^{1 / 2}}{\left[\gamma\left\{1-\frac{c^{2}}{\beta_{a}^{2}}\right\}\right]^{1 / 2}}
$$

which for an initial stress-free, isotropic, homogeneous, elastic layer (i.e. $\left.\gamma=1, N=L=\mu^{\prime}\right)$ lying over an initial stress-free, isotropic, homogeneous half-space (i.e. $\xi=0, \mathrm{~b}=0, \mathrm{a}=0$ ) is reduced to

$$
\frac{\mu^{\prime}}{\mu_{0}} \frac{\sqrt{\left(1-\frac{c^{2}}{c_{\beta}^{2}}\right)}}{\sqrt{\left(\frac{c^{2}}{c_{o s}^{2}}-1\right)}}=\tan \left\{K H \sqrt{\left(\frac{c^{2}}{c_{\beta}^{2}}-1\right)}\right\} .
$$

Eq. (8) gives the phase speed $\mathrm{c}$ of Love wave in a homogeneous layer over a homogeneous half-space when the upper boundary plane of the layer is assumed to be rigid.

Note that the equation for the phase speed c of Love waves in a layer overlaying a half-space, when the upper boundary plane is not rigid, is given in Ewing et al. [12]

$$
\frac{\mu_{0}}{\mu^{\prime}} \frac{\sqrt{\left(1-\frac{c^{2}}{c_{o s}^{2}}\right)}}{\sqrt{\left(\frac{c^{2}}{c_{\beta}^{2}}-1\right)}}=\tan \left\{K H \sqrt{\left(\frac{c^{2}}{c_{\beta}^{2}}-1\right)}\right\}
$$

It can be seen from Eqs. (8) and (9) that the phase speed of Love waves in a layer with a rigid surface is different from that in a layer with a free surface.

\section{NUMERICAL CALCULATIONS AND DISCUSSION}

To study the effect of porosity, anisotropy, nonhomogeneity and initial stresses on the propagation of Love waves under rigid layer, numerical calculations have been performed with different values of the parameters representing the above characteristics from Eq. (4). For these 
computations we have taken $\mu_{0} / L=2.5$ and $\beta_{a} / c_{o s}=0.7$ and the results are presented in figures 2-5. All these figures we have shown the variation of dimensionless velocity $c / \beta_{a}$ against dimensionless wave number $K H$.

\section{TABLE I: CURVES FOR DIFFERENT VALUES OF D}

\begin{tabular}{|l|l|}
\hline Curve no. & $d$ \\
\hline 1 & 0.6 \\
\hline 2 & 0.7 \\
\hline 3 & 0.8 \\
\hline 4 & 0.9 \\
\hline 5 & 1.0 \\
\hline
\end{tabular}

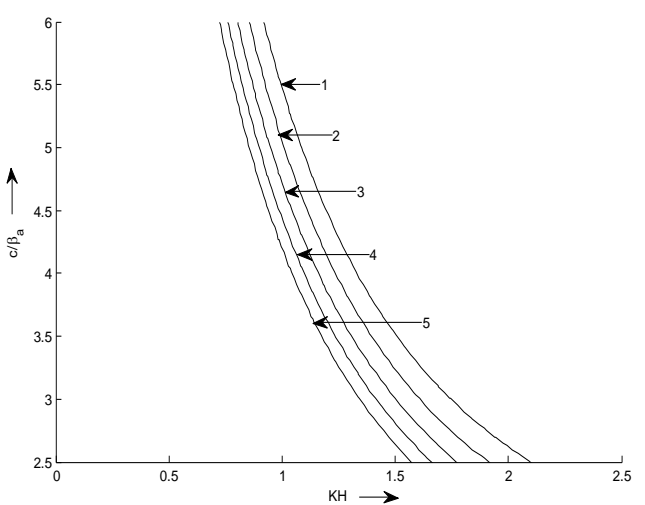

Fig. 2. Waves form.

$$
\gamma=1, \frac{b}{k}=0.4, \frac{a}{k}=1, \xi=0 .
$$

TABLE II: CURVES FOR DIFFERENT VALUES OF Y AND D

\begin{tabular}{|l|l|l|}
\hline Curve no. & $\gamma$ & $\mathrm{d}$ \\
\hline 1 & 1 & 1 \\
\hline 2 & 1 & 0.6154 \\
\hline 3 & 2 & 0.6154 \\
\hline
\end{tabular}

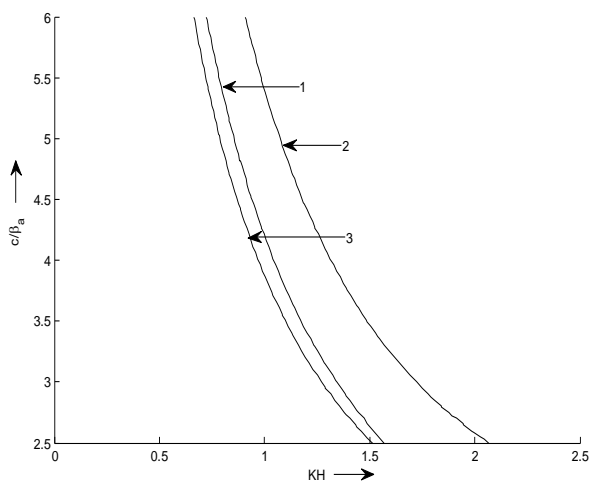

Fig. 3. The effect of anisotropy on the propagation of Love waves in a porous medium.

Fig. 2 shows the effect of porosity on the propagation of Love waves under rigid layer in a nonhomogeneous, anisotropic, initially stress free medium. We have plotted the curves for different values of $d$ as shown in Table I. It has been observed that as the porosity increases (i.e. as the value of $d$ decreases), the phase velocity of Love wave increases.
The effect of anisotropy on the propagation of Love waves in a porous medium is shown in Fig. 3. It has been observed that as the anisotropicity increases, the velocity of Love waves in the porous medium decreases.

Velocity dispersion curves of Love waves in a porous layer for various porosity in terms of parameter $d$ and for effect of anisotropy $(\gamma)$ in the porous layer on the propagation of Love waves for $\gamma=1, \frac{b}{k}=0.4, \frac{a}{k}=1, \xi=0$.

\section{CONCLUSION}

We can conclude that the phase velocity of Love waves is considerably influenced by rigidity, porosity and anisotropy of the porous medium, inhomogeneity of the half-space and prestressing in both the media. The effect of these characteristics of the media on the phase velocity of Love waves has been discussed and compared with the classical case when both the media are homogeneous, isotropic and initially stress.

\section{REFERENCES}

[1] A. E. H. Love, A treatise on mathematical theory of elasticity, New York: Dover Publication, 1944.

[2] M. A. Biot, "Theory of elasticity and consolidation for a porous anisotropic solid," J. Appl. Phys., vol. 26, pp. 182, 1955.

[3] M. A. Biot, "Theory of deformation of a porous viscoelastic anisotropic solid, J. Appl. Phys., vol. 27, pp. 459, 1956.

[4] M. A. Biot, "Theory of propagation of elastic waves in fluid saturated porous solid," J. Acoust. Soc. Am., vol. 8, pp. 168, 1956.

[5] M. A. Biot, "Mechanics of deformation and acoustic propagation in porous -media," J. Appl. Phys., vol. 33, pp. 1482, 1962

[6] M. A. Biot, Mechanics of incremental deformation, New York: John Wiley and Sons Inc., 1965.

[7] S. K. Bose, "Wave propagation in marine sediments and water saturated soils," Pure Appl. Geophys., vol. 52, pp. 27-40, 1962.

[8] R. Burridge and C. A. Vargas, "The fundamental solution in dynamic poro-elasticity,” Geophys. J. R. Astron. Soc., vol. 58, pp. 61-90, 1979.

[9] A. Chattopadhyay and R. K. De, "Love type waves in a porous layer with irregular interface," Int J. of Engg. Sci., vol. 21, no. 11, pp. 1295 1983.

[10] J. L. Nowinski, The effect of high initial stress on the propagation of Love wave in an isotropic elastic incompressible medium, some aspects of Mechanicsof continua, (a book dedicated as a tribute to the memory of Prof. B. Sen), part I, pp. 14-28, 1977.

[11] W. H. Weiskopf, "Stresses in soils under a foundation," J. Franklin Inst., vol. 239, pp. 445-465, 1945.

[12] W. M. Ewing, W. S. Jardetzky, and F. Press, Elastic waves in layered Media, New York: Mcgraw Hill, 1957.

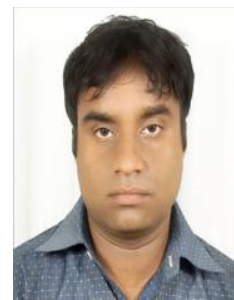

S. Kundu obtained his M.Sc. (Maths \& Computing) and M.Phil. (Applied Mathematics) from Indian School of Mines, Dhanbad. He received his Ph.D. in Applied Mathematics from Indian School of Mines, Dhanbad. He is currently working as an Assistant Professor in the department of Applied Mathematics, Indian School of Mines, Dhanbad, Jharkhand, India. He has several publications in International journals. He has also presented several papers in International and National conferences. 